\title{
Multimedia Content Delivery for Emerging 5G-Satellite Networks
}

\author{
Giuseppe Araniti, Igor Bisio, Mauro De Sanctis, Antonino Orsino, and John Cosmas
}

\begin{abstract}
Multimedia content delivery over satellite systems is considered as a promising service in emerging $5 \mathrm{G}$ networks. The aim of this paper is to design a novel radio resource management algorithm for efficiently managing multicast multimedia content transmission over satellite network. The proposed approach performs the spectrum management on a per-group basis, by splitting multicast terminals into different subgroups according to the experienced channel qualities. We demonstrate that subgrouping policy defined by the authors as multicast subgrouping-maximum satisfaction index (MS-MSI), based on a new metric (i.e., MSI), overcomes the weakness of the previous techniques proposed in the literature and provides the best tradeoff between user throughput and fairness. As a further result, we demonstrate that MS-MSI is robust to the long propagation delay of satellite links. An extensive simulation campaign has been conducted by considering several satellite environments.
\end{abstract}

Index Terms-LTE-satellite, multicast, resource allocation, Pérez-Fontán channel model, 5G.

\section{INTRODUCTION}

$\mathbf{N}$ OWADAYS, a growing number of people expect to access the same services they have at home even while traveling on cruise liners, flights, high-speed trains and vehicles. This ubiquitous coverage could be achieved by extending the emerging fifth generation $(5 \mathrm{G})$ networks with the satellite's wide coverage. The role of satellite in $5 \mathrm{G}$ networks is still under discussion [1]; nevertheless, the scientific community is addressing how to exploit the emerging Long Term Evolution (LTE)-Satellite architecture [2], [3] as a possible starting point for the 5G-Satellite definition [1].

A further $5 \mathrm{G}$ system challenge is to identify key technologies for offloading the network traffic in order to manage the huge demand of multimedia services. One of the possible technologies is identified as Device-to-Device (D2D) communications [4]. This new paradigm allows the direct transmission among nearby users thereby assuring high data

Manuscript received June 17, 2015; revised October 7, 2015 and November 20, 2015; accepted December 3, 2015. Date of publication January 14, 2016; date of current version March 2, 2016.

G. Araniti and A. Orsino are with the ARTS Laboratory, DIIES Department, University Mediterranea of Reggio Calabria, Reggio Calabria 89100, Italy (e-mail: araniti@unirc.it; antonino.orsino@unirc.it).

I. Bisio is with the DITEN Department, University of Genoa, Genoa 16100, Italy (e-mail: igor.bisio@unige.it).

M. De Sanctis is with the University of Rome Tor Vergata, Rome 00173, Italy (e-mail: mauro.de.sanctis@uniroma2.it).

J. Cosmas is with the WNCC, Brunel University, Uxbridge UB8 3PH, U.K. (e-mail: john.cosmas@brunel.ac.uk).

Color versions of one or more of the figures in this paper are available online at http://ieeexplore.ieee.org.

Digital Object Identifier 10.1109/TBC.2015.2511625 rate, low latency and higher energy efficiency. Nevertheless, the increased number of D2D pairs within a network coverage could lead to problems in terms of interference and management of radio resources [5]. In fact, the level of interference increases linearly with the number of users that want to communicate each other since D2D transmissions mostly are made by reusing the radio spectrum of cellular users [6]. As a consequence, the radio resource management process becomes complex and, in some cases, difficult to manage.

To overcome such issues, satellite networks could be a promising candidate for off-loading the terrestrial $5 \mathrm{G}$ networks traffic. In particular, significant advantages are expected for bandwidth-hungry services such as video, where the broadcast and multicast capabilities of a satellite system could be efficiently exploited. Indeed, video content delivery is one of the fastest growing services [7], especially over satellite networks; it is expected that by 2018 the video carried by wireless networks will be 8 times larger than it is in 2014 [8], reaching 11 exabytes [9]. Given the high interest in multimedia transmissions towards multiple destinations, the 3rd Generation Partnership Project (3GPP) defined a solution to deliver multicast and broadcast services over wireless networks namely Multimedia Broadcast Multicast Service (MBMS) [10]. Since 3GPP Release 8, MBMS has been extended to the Long Term Evolution (LTE) standard and it is now called evolved MBMS (eMBMS) [11].

This paper focuses on the Radio Resource Management (RRM) of multicast services in LTE-Satellite environments for providing video contents, where the RRM has to be performed on a per-group basis, since a group of users is simultaneously served by the satellite with one single radio transmission. As a consequence, the selection of transmission parameters (i.e., modulation and coding scheme, MCS) has to take into account the channel qualities of all involved multicast members.

Traditional approaches like conservative and opportunistic multicasting schemes [12] suffer from inefficiencies in terms of poor spectrum efficiency and inadequate short-term fairness, respectively. A promising RRM scheme for satellite multicast environments is subgrouping. It serves all multicast terminals in every time slot by splitting them into different subgroups according to the experienced channel qualities. This improves the video session quality compared to other strategies [13]. An example can be found in [14], where multicast subgrouping policies, which are based on maximum throughput (MT) and proportional fair (PF) metrics, are compared with a novel metric defined by the authors as multicast subgrouping-minimum 
dissatisfaction index (MDI). By analyzing the results in [14], we observed the impossibility of having a RRM strategy that outperforms all other policies in all considered metrics; this makes it very hard to define in an effective way which is the most performing multicast subgrouping strategy.

The intended contribution of our paper is to extend the study we conducted in [14] in order to demonstrate that subgrouping approach, based on a metric named multicast subgroupingmaximum satisfaction index (MS-MSI), overcomes the weakness of the previous techniques and allows to efficiently deliver multimedia content in the emerging satellite system. In addition to the previous study [14], we extend the analytical model for supporting multicast video content delivery. We consider a scalable video coding technique where a minimum data rate is guaranteed to all the multicast groups whereas additional enhancement layers are conveyed to a subset of users with good channel conditions. As a further improvement, we exploit an effective technique, which allows to measure the overall performance of multicast RRM strategies through a single mark, thus guaranteeing to effectively define the policy with the best overall performance. This result is obtained by solving a Multi-Criteria Decision-Making (MCDM) problem. As a further contribution, we investigated the effects of subgrouping approaches (already designed and well investigated for terrestrial system in our paper [15] and in our referred works) in a satellite environment. In particular, we conducted a simulation campaign for demonstrating that the subgrouping approach is able to maintain the same performance achieved for terrestrial systems whereas the opportunistic approach is delay sensitive and thus cannot be exploited for multimedia content delivery in satellite environment, because it could require a high number of retransmissions. Furthermore, we handle the complexity burden of multicast subgroup formation. In fact, the selection of the optimal subgroup configuration based on an exhaustive search scheme (ESS) or global solvers (as in [14]), introduces a high load and limits the effectiveness of subgrouping in practical satellite systems. For this reason, we propose an approach for drastically decreasing the time required for resource allocation. Finally, we consider several satellite environments in order to generalize the obtained results. The simulation campaign shows how MS-MSI provides better overall performance when delivering video content compared to both state-of-the-art multicast solutions and the novel policies based on sub-grouping that employ maximum throughput and proportional fair approaches. Moreover, the obtained results demonstrate that MS-MSI is robust to the long propagation delay of satellite links, since all the multicast members are able to decode without errors the data contents transmitted by the satellite.

The remainder of the paper is organized as follows. In Section II the related works are discussed and in Section III the reference S-LTE system model with service configuration is described. The subgroup formation and the RRM policies are described in Section IV, whereas the performance evaluation settings, the adopted MCDM algorithm, and the results are summarized in Section V. Finally, concluding remarks are given in Section VI.

\section{RELATED WORK}

One of the most investigated issues on multicasting over Orthogonal Frequency Division Multiple Access (OFDMA) systems is related to the link adaptation procedures [16], also known as Adaptive Modulation and Coding (AMC) schemes. The AMC is expected to guarantee meaningful improvements also in satellite networks [17]. Indeed, in a multicast scenario where several destinations require the same data, the setting of transmission parameters have to be performed on a per-group basis. This involves several issues mainly related to different channel conditions experienced by multicast group members within a spotbeam [16]. Generally, the terminals with good channel conditions can support high MCS levels, ${ }^{1}$ whereas the ones undergoing a bad channel have to be served with more robust MCSs.

In such a direction, several approaches have been proposed in the literature. In the conservative approach [13], named here conventional multicast scheme (CMS), the whole set of destinations is served by adapting the MCS levels to those supported by the user experiencing the worst channel conditions. Although this technique guarantees perfect fairness, since resources are evenly distributed and all multicast members experience the same data rate, it suffers from poor spectral efficiency.

To overcome this limitation, the opportunistic approach [16] endeavors to serve, in any given time slot, only a portion of multicast users in order to maximize a given objective function, such as system throughput. The goal of this approach, named Multicast Link Adaptation (MLA) [12], is to exploit the multi-user diversity in the resource allocation process, although it may limit the multicast gain, i.e., the number of users successfully served in each time slot. As a consequence, additional data coding (e.g., rateless codes) is required for ensuring the users keep the transmitter informed of which portion of file is received. Although opportunistic approaches can achieve long-term fairness (which can be considered suitable in applications such as file delivery), it cannot achieve short-term fairness (since not all users are served within every time slot) which, conversely, is more important in streaming applications [16].

To reduce the bottleneck effects of conservative and opportunistic schemes influenced by users in poor channel conditions, a promising RRM approach for multicast environments is represented by the subgrouping [14]. The goal of this technique is to split the entire multicast group into smaller sub-groups in order to serve them every Transmission Time Interval (TTI) slot by guaranteeing improvements in terms of session quality and user satisfaction. A subgrouping scheme, based on a metric named Minimum Dissatisfaction Index (MDI), has been proposed by the authors in [14]. The aim of this new metric is to guarantee a feasible level of fairness without decreasing in an evident manner the user throughput.

Nevertheless, from the analysis proposed in [14] it is not possible to define which is the most suitable subgrouping technique in order to provide a good trade-off in terms

\footnotetext{
${ }^{1}$ The admissible throughput values per MCS level are set according to Table 7.1.7.2.1-1 in [18].
} 
of throughput and fairness. For solving the aforementioned issue, in this paper we adopt a multi-criteria decision making (MCDM) approach based on the technique for order of preference by similarity to ideal solution (TOPSIS) [19].

Many MCDM techniques have been proposed in the literature. Some of these techniques are based on weighted point method [20], matrix approach [21], analytic hierarchy process (AHP) [22] and analytic network process (ANP) [23]. However, most of these methods are developed with respect to definitive data, without taking into account several factors, such as imprecise preferences, additional qualitative criteria and incomplete information. TOPSIS, instead, is applied to solve these issues by providing numerous advantages: (i) the processing of TOPSIS fits the human decision selection process; (ii) the best and the worst solutions are compared quantitatively; (iii) the algorithm is easy to implement.

The long delay introduced by the satellite link [3], [13] is a further challenging issue that in satellite environments should not be neglected. The fast link adaptation process has to be sensitive to the instantaneous variations of user channel conditions. For this reason, the misalignment between the channel quality feedback transmitted to the network and the channel quality experienced by the mobile users at the reception of data traffic is a key component of the S-LTE network. Therefore, new techniques of channel quality prediction have to be investigated to improve the efficiency of link adaptation. Many works in the literature deal with the prediction problem [24]. Among those, in this paper we adopt the Autoregressive Integrated Moving Average Model (ARIMA) [25] which is characterized by a simple implementation and guarantees high accuracy. The aforementioned conservative (i.e., CMS) and opportunistic (i.e., MLA) approaches are evaluated as benchmark in the Section V.

\section{RefEREnce Scenario AND System Model}

\section{A. The Satellite-LTE Scenario}

As mentioned in Section I, the satellite-LTE architecture [2], [3], [26] will play an important role in the definition of 5G satellite network [1]. In Fig. 1, we depict an enhanced S-LTE architecture designed for supporting multicast transmissions. In particular, it is composed of a GEO satellite (hereinafter S-LTE), equipped with a S-LTE air interface [2], [3] that communicates on one side with the S-LTE terminals and on the other side with an LTE ground component that performs the radio access procedures [14]. In particular, the ground component is the Satellite eNodeB (S-eNodeB), which handles the configuration of physical layer parameters, e.g., the MCS of the S-LTE radio interface.

The GEO satellite in our scenario is used as a transparent transponder and does not include any on-board processing equipment or additional components with respect to the standard usage. Since in our work we are interested in multicasting transmission, multicast data are transmitted through the forward link (i.e., S-eNodeB $\rightarrow$ S-LTE $\rightarrow$ S-LTE terminals) whereas the reverse link (i.e., S-LTE terminals $\rightarrow$ S-LTE $\rightarrow$ $\mathrm{S}$-eNodeB) is exploited for transmitting the user signalling information.

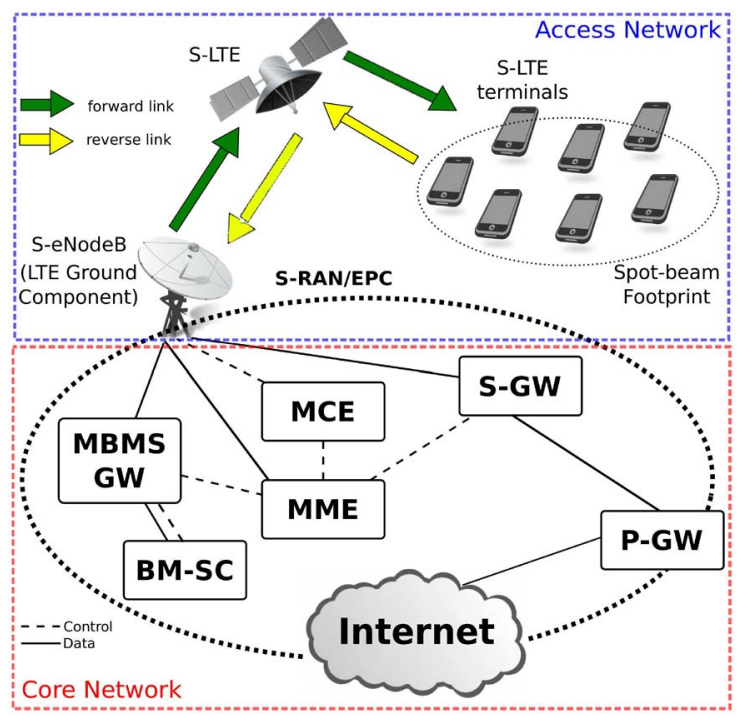

Fig. 1. The Satellite-LTE reference scenario.

Hence, the propagation delay is considered to be the time the signal takes to travel from the S-eNobeB to the S-LTE terminals where the signal encounters an uplink and downlink transmission, and vice versa. Therefore, to evaluate numerically the propagation delay we have to consider that the speed of the light is around $300.000 \mathrm{~km} / \mathrm{sec}$ and that the altitude of the satellite is $35.838 \mathrm{~km}$. Then the communication delay between two ground terminals/stations (through the satellite) is about $(2 \cdot 35.838) / 300,000=0.24 \mathrm{sec}$. However, if the receiver is not perfectly under the satellite, the delay to transmit a signal to the satellite is close to $0.27 \mathrm{sec}$. Therefore, in two-way communications (in our case, for exchanging signalling and transmission parameters) the propagation delay of the total Round Trip Time (RTT) is typically equal to $0.54 \mathrm{sec}$. In this calculation we do not consider other types of delays, i.e., transmission delay, processing delay, which are negligible with respect to the propagation delay [3].

In architectures such as this, group-oriented services are handled through the eMBMS standard. The MultiCell/Multicast Coordination Entity (MCE) is used for the adaption of transmission parameters in case of multicast transmission involving different S-eNodeB. The MBMS Gateway (MBMS-GW) is a logical entity whose principal function is data packets' forwarding to S-eNodeBs while the Broadcast Multicast-Service Center (BM-SC) is the MBMS traffic source which also accomplishes service announcement and group membership functions.

The S-eNodeB is connected to the core network by means of the $S 1$ interface, namely: the $S 1-u$ link to the Serving Gateway (S-GW) and Packet Data Network Gateway (P-GW) and the S1-c link to the Mobility Management Entity (MME) [3]. Multiple S-eNodeBs are linked with each other and with the $X 2$ interface in order to support the active-mode mobility. This interface is also used for further functions, such as the interference cancellation techniques (i.e., ICIC) and to support mobility between neighboring beams' coverage areas. 
TABLE I

NOTATIONS USED IN THE PAPER

\begin{tabular}{ll}
\hline$N$ & Number of RBs \\
$K$ & Multicast group size \\
$e_{k}$ & Multicast member $k$ \\
$M$ & Number of admissible CQI levels \\
$\ell_{k}$ & CQI of user $k$ \\
$b_{m}^{M I N}$ & Minimum data rate achieved by one RB with $m$-th MCS \\
$B_{m}^{M A X}$ & Maximum data rate achieved by all RB with $m$-th MCS \\
$b_{v i d e o}^{M I N}$ & Minimum data rate requested by video application \\
$B_{\text {video }}^{M A X}$ & Maximum data rate requested by video application \\
$S_{m}$ & Number of multicast subgroups \\
$u_{m}$ & Number of multicast users served with the $m$-th MCS \\
$r_{m}$ & Number of RBs assigned to the subgroup with the $m$-th MCS \\
\hline
\end{tabular}

The S-LTE air interface exploits OFDMA in downlink direction, where the available spectrum is split into several sub-carriers spaced of $15 \mathrm{kHz}$. The resource allocation is performed in terms of Resource Blocks (RBs), each one spanning 12 adjacent sub-carriers and lasting $0.5 \mathrm{~ms}$. The number of RBs for data transmission can vary from 6 to 100, according to the channel bandwidth deployment [2]. The RRM is in charge for performing the link adaptation procedures in order to dynamically adapt the transmission parameters, i.e., MCSs, with the aim of exploiting the greatest potential of OFDMA. Every TTI, which lasts $1 \mathrm{~ms}$, the RRM allocates the resources on a RB-pair basis according to the Channel Quality Indicator (CQI) feedback sent by the S-LTE terminals. The CQI is an indication of the maximum MCS that guarantees a successful data reception according to the channel conditions experienced by the terminal.

We assume that the communication channel is not affected by nonlinear distortions. This can be considered as a realistic assumption because: $(i)$ the transmission band of interest is relatively low (i.e., S-band) therefore current power amplifiers in this band can be driven in a fully linear region at the expense of a low power efficiency, and (ii) several variants of OFDMA exist which have a low sensitivity to non-linear distortions because of a lower Peak-to-Average Power Ratio (PAPR), such as Single Carrier FDMA (SC-FDMA) and Constant Envelope SC-FDMA (CE-SC-FDMA) [27].

\section{B. Proposed Sub-Group Policy and Design Aspects}

In this Section we firstly introduce the notations used in the paper and then we present the proposed subgroup-based algorithm.

Let $N$ be the number of RBs available for the transmission of a satellite multicast stream. Such stream is transmitted towards $K$ multicast members, denoted with $e_{1}, e_{2}, \ldots, e_{K}$. Each user performs the channel estimation by calculating the CQI, which is transmitted at the S-eNodeB in order to perform the RRM procedures. We indicate with $M$ the number of admissible CQI levels; we further denote with $\ell_{k} \in\{1,2, \ldots, M\}$ the CQI reported by member $e_{k}$, for $k=1,2, \ldots, K$. Each CQI level is associated with a given supported MCS. Accordingly, we indicate with $b_{m}^{M I N}$ (where $m=1, \ldots, M)$ the data attained when one RB is transmitted according to the MCS corresponding to the $m$-th CQI. Similarly, let $B_{m}^{M A X}$ be the maximum data rate for the $m$-th
MCS level, achieved when all $N$ RBs are assigned. Since in this work we consider a scalable video coding technique in order to guarantee a minimum data rate to all the multicast members, we define with $b_{\text {video }}^{M I N}$ and $B_{\text {video }}^{M A X}$ the minimum and the maximum data rate requested by the video application, respectively.

The proposed RRM policy aims at splitting the users into $S$ multicast subgroups and at opportunely distributing the available RBs according to the collected CQI values. The proposed subgroup-based scheme foresees two phases.

1) CQI Collection: The S-eNodeB collects the CQI feedbacks from each of the S-LTE terminals belonging to the same multicast group (i.e., $\ell_{k}$, with $k=1,2, \ldots, K$ ).

2) Subgroup Creation: Based on the users' CQI feedback values, the proposed RRM algorithm determines the subgroup configuration. A subgroup configuration is denoted by the number of subgroups $S$ to enable, the related MCSs, and the $\mathrm{RBs}$ that are to associate to each subgroup. Under the assumption that each subgroup is characterized by a different MCS, the number of subgroups $S$ varies from 1 to $M$. We assume that all S-LTE terminals with the same CQI value are associated to the same subgroup, although the same subgroup may include S-LTE terminals with different CQI values.

The subgroup formations are performed with the following objectives: (i) maximizing the system capacity with the constraint that each multicast member can successfully demodulate the received signal (i.e., the MCS associated to a given subgroup must be supported by all users belonging to such a subgroup); (ii) optimize a given objective function.

We denote with $\mathcal{U}=\left\{u_{1}, u_{2}, \ldots, u_{M}\right\}$ the final subgroup configuration, where $u_{m}$ represents the number of S-LTE terminals assigned to an MCS corresponding to the $m$-th CQI, and with $\mathcal{R}=\left\{r_{1}, r_{2}, \ldots, r_{m}\right\}$ the distribution of the resources assigned to the subgroup configuration. If $r_{m} \in \mathcal{R}$ is greater than zero, then the subgroup related to the $m$-th CQI level is enabled (that is $u_{m} \neq 0$ ) and $r_{m}$ represents the number of resources allocated to the subgroup. If $r_{m}=0$ such a subgroup is not enabled. The number $S$ of enabled subgroups is thus given by the sum of items $r_{m} \in \mathcal{R}$ greater than zero. Depending on the amount of the RBs given to a subgroup, all the S-LTE terminals of the subgroup $m$ will be served with a data rate given by:

$$
b_{m}^{T H}=\left\{\max \left(b_{i}^{M I N} r_{i}\right), i=1,2, \ldots, m\right\}
$$

The characterization of $b_{m}^{T H}$ also guarantees that the data rate achieved by each S-LTE terminal in the subgroup is under the value of $B_{m}^{M A X}$.

To describe our model, we introduce the binary variables $x_{k, m}, k=1, \ldots, K, m=1, \ldots, m$, such that:

$$
x_{k, m}= \begin{cases}1, & \text { if } e_{k} \text { is assigned to the } m \text {-th MCS } \\ 0, & \text { otherwise }\end{cases}
$$

The subgroup formation problem can be written, in a general form, as follows:

$$
\boldsymbol{\Pi}=\underset{r_{m}, x_{k, m}}{\arg \max }\left\{\sum_{m=1}^{M} \phi\left(b_{m}^{T H}\right) \sum_{k=1}^{K} x_{k, m}\right\}
$$




$$
\begin{array}{lll}
\text { s.t. } & \sum_{m=1}^{M} r_{m}=N & \\
& r_{m} \in\{0,1, \ldots, N\}, & m=1, \ldots, M \\
& \sum_{m=1}^{\ell_{k}} x_{k, m}=1, \quad \sum_{m=\ell_{k}+1}^{M} x_{k, m}=0, & k=1, \ldots, K \\
& x_{k, m} \in\{0,1\}, \quad k=1, \ldots, K, \quad & m=1, \ldots, M \\
& \frac{1}{K} \sum_{k=1}^{K} x_{k, m} \leq r_{m} \leq N \sum_{k=1}^{K} x_{k, m}, & m=1, \ldots, M
\end{array}
$$

In eq. (3), $\phi\left(b_{m}^{T H}\right)$ indicates a generic objective function, which depends on the amount of RBs and by the MCSs available in the system. The constraint (4a) guarantees that the whole RB set is exploited by the enabled subgroups (i.e., all the users are served with the minimum CQI). The constraints (4c) take into account the initial configuration, so that in the new one each user is associated to one subgroup only, with the additional requirement that the related MCS level must be less than or equal to the MCS level corresponding to the CQI initially reported. All considered constraints (4a)-(4e) require that:

$$
\begin{cases}r_{m}=0, & \text { if } \sum_{k=1}^{K} x_{k, m}=0 \\ 1 \leq r_{m} \leq N, & \text { if } \sum_{k=1}^{K} x_{k, m} \geq 1\end{cases}
$$

i.e., a nonzero $\mathrm{RB}$ value is assigned only to subgroups with at least one user. In addition, the data rate $b_{m}^{T H}$ is properly set according to the QoS requirements of the multicast service and is bounded by:

$$
b_{\text {video }}^{\text {MIN }} \leq b_{m}^{T H} \leq B_{\text {video }}^{M A X},
$$

in order to guarantee the minimum data rate to all the multicast members. In particular, when $B_{\text {video }}^{M A X}<B_{m}^{M A X}$ the saved bandwidth could be used for providing further services.

\section{Subgroup Formation Procedures}

\section{A. Approaches for Subgroup Formation}

In this Section, we consider three approaches designed for subgroup formation in the considered satellite environment: (i) the multicast subgrouping maximum throughput; (ii) the multicast subgrouping proportional fairness; (iii) the multicast subgrouping maximum satisfaction index.

1) Multicast Subgrouping Maximum Throughput (MS-MT): The Multicast Subgrouping Maximum Throughput (MS-MT) algorithm is based on the maximization of the sum of the data rates experienced by all the multicast members. Hence, the maximization problem, which is based on the MS-MT approach tailored for the addressed satellite subgrouping scenario, can be expressed as follows:

$$
\mathbf{\Pi}^{\mathbf{M S}-\mathbf{M T}}=\underset{r_{m}, x_{k, m}}{\arg \max }\left\{\sum_{m=1}^{M} b_{m}^{T H} \sum_{k=1}^{K} x_{k, m}\right\}
$$

subject to constraints (4a)-(4e), (5), and (6).
2) Multicast Subgrouping Proportional Fair (MS-PF): The aim of a Multicast Subgrouping Proportional Fair (MS-PF) resource allocation is to improve the fairness among multicast destinations while increasing the throughput. As shown for instance in [28], a MS-PF resource allocation can be obtained through the maximization of the sum of the logarithm of user data rates. In the addressed satellite subgrouping scenario, according to [28], the MS-PF optimization problem can be written as:

$$
\mathbf{\Pi}^{\mathbf{M S}-\mathbf{P F}}=\underset{r_{m}, x_{k, m}}{\arg \max }\left\{\sum_{m=1}^{M} \log \left(b_{m}^{T H}\right) \sum_{k=1}^{K} x_{k, m}\right\}
$$

subject to constraints (4a)-(4e), (5), and (6).

3) Multicast Subgrouping Maximum Satisfaction Index (MS-MSI): The RRM policy, called Multicast Subgrouping Maximum Satisfaction Index (MS-MSI), is based on the optimization of a novel objective function conceived to guarantee an increased throughput with respect to the PF policy whilst do not significantly affecting the fairness among the multicast members. This goal is achieved through the maximization of the user satisfaction. In particular, we define the User Dissatisfaction Index (UDI) for a generic multicast member $e_{k}$ as follows:

$$
\omega_{k}=\frac{B_{m_{k}}^{e f f}-\sum_{m=1}^{M} b_{m}^{T H} x_{k, m}}{B_{m_{k}}^{e f f}}
$$

i.e., $\omega_{k}$ measures the difference between the maximum data rate supported by the S-LTE terminal according to the experienced channel conditions, i.e., $B_{m_{k}}^{e f f}=\min \left(B_{m_{k}}^{M A X}, B_{\text {video }}^{M A X}\right)$, and the data rate assigned to the associated subgroup. According to (9), such a difference is normalized on $B_{m_{k}}^{e f f}$ in order to maintain the fairness among multicast destinations. As a consequence, $\omega_{k} \in[0,1]$. In detail, the minimum dissatisfaction, i.e., $\omega_{k}=0$, is achieved when the assigned data rate is equal to the maximum allowable one, i.e., the subgroup of the multicast terminal is served with the MCS supported by the terminal and the resources are assigned to such a subgroup in order to guarantee $B_{m_{k}}^{M A X}$.

The proposed MS-MSI is designed in order to exploit a novel objective function, namely the Maximum Satisfaction Index (MSI), which represents the reverse of the average UDI over the whole set of S-LTE terminals. According to this definition, the objective function for multicast subgroup formation can be described as follows:

$$
Q^{M S I}=\left(1-\frac{1}{K} \sum_{k=1}^{K}\left(\frac{B_{m_{k}}^{e f f}-\sum_{m=1}^{M} b_{m}^{T H} x_{k, m}}{B_{m_{k}}^{e f f}}\right)\right)
$$

aimed at selecting the best subgroup configuration that maximize the MSI to address both fairness and throughput issues. Then, eq. (10) can be recast in the form:

$$
Q^{M S I}=\frac{1}{K}\left(\sum_{m=1}^{M} b_{m}^{T H} \sum_{k=1}^{K} \frac{x_{k, m}}{B_{m_{k}}^{e f f}}\right)
$$


TABLE II

COMPARISON OF ESS AND OSS SCHEMES

\begin{tabular}{ccc}
\hline Number of RBs & \multicolumn{2}{c}{ Number of Configurations } \\
& ESS & OSS \\
\hline 2 & $3 \cdot 10^{4}$ & $1.2 \cdot 10^{2}$ \\
4 & $1 \cdot 10^{9}$ & $2.6 \cdot 10^{3}$ \\
6 & $4.7 \cdot 10^{11}$ & $2.2 \cdot 10^{4}$ \\
8 & $3.5 \cdot 10^{13}$ & $1 \cdot 10^{5}$ \\
10 & $1 \cdot 10^{15}$ & $3.2 \cdot 10^{5}$ \\
12 & $1.5 \cdot 10^{16}$ & $8.1 \cdot 10^{5}$ \\
14 & $1.6 \cdot 10^{17}$ & $1.8 \cdot 10^{6}$ \\
15 & $4.4 \cdot 10^{17}$ & $2.7 \cdot 10^{6}$ \\
\hline
\end{tabular}

Hence, the MS-MSI optimization problem can be written as follows:

$$
\boldsymbol{\Pi}^{\mathbf{M S}-\mathbf{M S I}}=\underset{r_{m}, x_{k, m}}{\arg \max }\left\{\frac{1}{K}\left(\sum_{m=1}^{M} b_{m}^{T H} \sum_{k=1}^{K} \frac{x_{k, m}}{B_{m_{k}}^{e f f}}\right)\right\}
$$

subject to constraints (4a)-(4e), (5), and (6).

\section{B. Complexity Cost Reduction}

One of the key issues of the proposed RRM algorithm is the search space definition and its related computational cost reduction. In particular, the computational time in order to perform the optimization problems presented in Sections IV-A1-IV-A3 are tightly related to the number of possible configurations to be analyzed. Based on the Exhaustive Search Scheme (ESS) [14], the computational cost is bounded by the number $\mathcal{R}$ of possible configurations when $N$ RBs are split among $M$ subgroups. In this case, the complexity cost become prohibitive and it is represented by a combination equal to $N^{M}$.

In this section we propose a solution aiming at reducing the search space (and, consequently, the computational costs), named Optimized Search Scheme (OSS). Considering two subgroups characterized by $M C S_{i}$ and $M C S_{j}$ in a generic configuration $\mathcal{R}$, the multicast subgrouping technique introduces a gain (in terms of spectral efficiency) if and only if the data rate of the subgroup with higher order $M C S$ (i.e., $b_{j}$ ) is higher compared to the rate of the subgroup with lower-order MCS (i.e., $b_{i}$ ). In particular, this condition can be characterized as follows:

$$
b_{j}>b_{i} \forall i, j: j>i, j \leq M, i=1, \ldots, j-1
$$

The idea proposed for the OSS approach, is to include in the space definition process all the candidate configurations that satisfy the conditions (13) and (4a). In this way, all the configurations that are not admissible for the solution of the problems (7), (8) and (12) are not evaluated in the space definition process. Due to this fact, the number of configurations generated by the OSS is significantly reduced with respect to the ESS scheme. These results are remarked in Table II which shows the overall configurations achieved by the two schemes (ESS and OSS) when the number of the RBs varies from 2 to 15 .

As expected, OSS allows to drastically reduce the configurations to be evaluated in the resource allocation when compared to the ESS. In particular, it can be noticed that this gain
TABLE III

Main Simulation Assumptions

\begin{tabular}{ll}
\hline Parameters & Value \\
\hline Frequency Bandwidth & S-2.618 GHz (S-Band) \\
Channel Bandwidth & $5 \mathrm{MHz}$ \\
Number of RBs & 25 \\
FFT size & 2048 \\
Sub-carrier Spacing & $15 \mathrm{kHz}$ \\
TTI & $1 \mathrm{~ms}$ \\
OFDM symbol duration & $83.33 \mu \mathrm{s}$ \\
Sampling interval & $32.55 \mathrm{~ns}$ \\
Cyclic Prefix Length & $16.67 \mu \mathrm{s}$ \\
UE distribuion & Uniform \\
Elevation & $40^{\circ}$ \\
S-LTE terminal speed & {$[3306090120] \mathrm{kmph}$} \\
\hline
\end{tabular}

increases when the number of RBs becomes large. Moreover, by exploiting the frequency-aggregated granularity introduced in LTE, OSS guarantees a reasonable search space dimension when the number of RBs is higher than 15 .

\section{Simulation Results}

\section{A. Simulation Scenario}

The simulation campaign has been carried out according to the guidelines defined in [26]. We addressed an application scenario consisting of two-way communications using multispot coverage with frequency reuse and Frequency Division Duplexing (FDD) scheme. Table III lists the main parameters related to the S-LTE scenarios.

According to [26], we developed a land-mobile satellite (LMS) channel simulator based on the Pérez-Fontán model [29]. This model considers a data set for the S-Band provided by Bradford University, U.K. [30]. The measurements have been conducted by placing the transmitter on board a plane whereas the receiver and the data acquisition system on a van rooftop. The plane flew parallel to the roadside at different elevations with respect to the receiver (i.e., $40^{\circ}, 60^{\circ}, 70^{\circ}$, and $80^{\circ}$ ). The receiver antennas utilised in the Pérez-Fontán studies have a hemispherical radiation pattern with a circular polarization. Moreover, the instantaneous velocity of the van was recorded and signal amplitude variations were measured through a logarithmic receiver.

Furthermore, the Pérez-Fontán model is based on the assumption of the existence on three different rates of change in the main propagation channel elements: the direct signal that may undergo shadowing/blockage effects and the multipath (specular and diffuse). These three rates of variation are described by means of a three-state Markov chain, a lognormal distribution and the coherent sum of the direct ray and the multipath echoes, respectively. In addition, we considered three propagation conditions: LOS, moderate shadowing and deep shadowing. Shadowing and multipath variations within each individual state are assumed to follow a Loo distribution [31]. The transitions between the propagation conditions are ruled by a 3-state Markov chain, whose initial probability vector $[\mathrm{W}]$ and transition probability matrix $[\mathrm{P}]$ have been derived from measurement data [29] and are provided for each of the considered scenarios (Table V). Therefore, the model allows to characterize the satellite channel in several environments and at several elevations. 


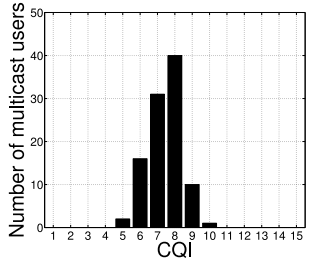

(a) Open

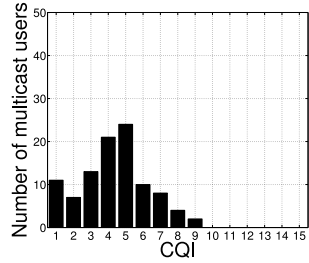

(b) Suburban

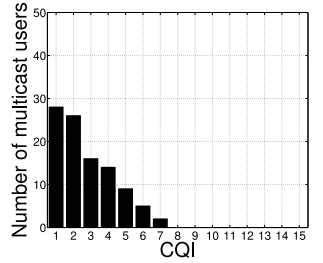

(c) Urban

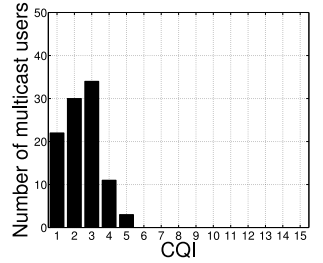

(d) Intermediate Tree Shadowed

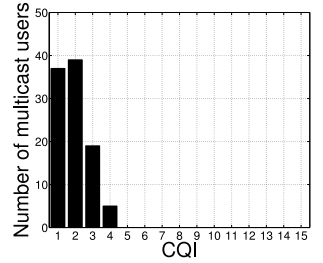

(e) Heavy Tree Shadowed

Fig. 2. Example of users' CQI distribution.

TABLE IV

CQI-MCS MAPPING [2]

\begin{tabular}{cccc}
\hline $\begin{array}{c}\text { CQI } \\
\text { index }\end{array}$ & $\begin{array}{c}\text { Modulation } \\
\text { Scheme }\end{array}$ & $\begin{array}{c}\text { Code rate } \\
\text { x 1024 }\end{array}$ & $\begin{array}{c}\text { Efficiency } \\
{[\mathrm{bit} / \mathrm{s} / \mathrm{Hz}]}\end{array}$ \\
\hline 1 & QPSK & 78 & 0.1523 \\
2 & QPSK & 120 & 0.2344 \\
3 & QPSK & 193 & 0.3770 \\
4 & QPSK & 308 & 0.6016 \\
5 & QPSK & 449 & 0.8770 \\
6 & QPSK & 602 & 1.1758 \\
7 & 16-QAM & 378 & 1.4766 \\
8 & 16-QAM & 490 & 1.9141 \\
9 & 16-QAM & 616 & 2.4063 \\
10 & 64-QAM & 466 & 2.7305 \\
11 & 64-QAM & 567 & 3.3223 \\
12 & 64-QAM & 677 & 3.9023 \\
13 & 64-QAM & 772 & 4.5234 \\
14 & 64-QAM & 873 & 5.1152 \\
15 & 64-QAM & 948 & 5.5547 \\
\hline
\end{tabular}

We exploited the described model because a measurement campaign specifically tailored for satellite-LTE network/devices does not yet exist. Nevertheless, we noticed that the measurement campaign conducted by Fontán et al. in [29] in S-band could be exploited as a first approximation in Satellite-LTE scenario without any losses of generality. In fact, we assumed that the mobile receivers are located in an outdoor environment with a receive antenna having a hemispherical radiation pattern. Therefore, the conditions of the Pérez-Fontán model are replicated, no matter if the antenna is located on a van rooftop or in a hand held satellite terminal.

Moreover, in this paper we considered the five environments depicted in Table V. Once the signal to noise ratio experienced by S-LTE terminals is obtained, it is subsequently mapped onto the respective CQI level which allows to receive the data transmission with a Block Error Rate (BLER) lower than 10\% [26]. In Table IV are listed the CQI values, with the related MCSs, considered in this paper. We assume the channel conditions do not to vary within a single TTI.

In the conducted simulation campaigns we assumed $K=$ 100 users joined in the multicast group. We considered an elevation angle of $40^{\circ}$ and the following environments: (i) Open; (ii) Suburban; (iii) Urban; (iv) Intermediate Tree Shadowed; (v) Heavy Tree Shadowed. For each considered environment, an example of users' CQI distribution is depicted in Fig. 2. In particular, the results provided in Fig. 2 show the number of multicast users that experience a certain level of CQI in a given TTI. For instance, if we consider the Heavy Tree Shadowed environment (i.e., Fig. 2(e)) we can see that, for a total number of 100 users: 36 users have a CQI equal to 1 ,
TABLE V

MARKov Chain Matrices [P]

\begin{tabular}{c|c|ccc}
\hline \hline Environment & $\mathrm{W}$ & \multicolumn{3}{c}{$\mathrm{P}$} \\
\hline \multirow{3}{*}{ Open } & 0.5 & 0.9530 & 0.0431 & 0.0039 \\
& 0.375 & 0.0515 & 0.9347 & 0.0138 \\
& 0.125 & 0.0334 & 0.0238 & 0.9428 \\
\hline \multirow{3}{*}{ Suburban } & 0.4545 & 0.8177 & 0.1715 & 0.0108 \\
& 0.4545 & 0.1544 & 0.7997 & 0.0459 \\
& 0.091 & 0.1400 & 0.1433 & 0.7167 \\
\hline \multirow{3}{*}{ Urban } & 0.4 & 0.8628 & 0.0737 & 0.0635 \\
& 0.2667 & 0.1247 & 0.8214 & 0.0539 \\
& 0.3333 & 0.0648 & 0.0546 & 0.8806 \\
\hline \multirow{3}{*}{ Intermediate Tree Shadowed } & 0.3929 & 0.7193 & 0.1865 & 0.0942 \\
& 0.3571 & 0.1848 & 0.7269 & 0.0883 \\
& 0.25 & 0.1771 & 0.0971 & 0.7258 \\
\hline \multirow{2}{*}{ Heavy Tree Shadowed } & 0.0 & 0.7792 & 0.0452 & 0.1756 \\
& 0.5 & 0 & 0.9259 & 0.0741 \\
& 0.5 & 0 & 0.0741 & 0.9259 \\
\hline \hline
\end{tabular}

39 users have a CQI equal to 2, 19 users have a CQI equal to 3 , and the reminder experience a CQI equal to 4 . If some CQI values do not have a corresponding number, then this means that no user experiences the given level of channel quality. Obviously, Figure 2 is a snapshot of the radio resource management performed during a single TTI whereas different values will be experienced by each user during the simulation time. It emerges that, as expected, the best channel conditions for multicast users are attained in the Open environment while, on average, multicast devices experience poorer qualities in other environments. In particular, the lowest CQI values are measured in the Heavy Tree Shadowed environment, where the maximum modulation supported by the users is QPSK.

As mentioned in the previous sections we compared the subgrouping approaches with the two policies: the CMS and the MLA [12]. Each simulation run has been repeated several times to get $95 \%$ confidence intervals.

The following simulation metrics are considered:

- Channel Data Rate (CDR). This parameter represents the amount of data transmitted over the radio channel, and consequently it is important for a well designed policy to achieve high CDR since this represents a performance of interest for the network provider.

- Aggregate Data Rate $(A D R)$. It indicates the sum of throughput values experienced by multicast users. The higher the ADR, the higher the throughput of multicast users.

- Jain's Fairness Index (JFI). The JFI is measured according to the well known formula in [32] and indicates the "fairness" in the distribution of throughputs experienced 
by multicast users [33], [34]. The JFI has values in $[1 / K, 1]$. The higher the JFI, the closer the throughput of multicast members.

- Multicast Normalized Throughput (MNT). The MNT is measured as the ratio between the CDR and the mean throughput measured among all multicast destinations. The MNT indicates how efficiently and fair the radio channel is exploited, i.e., a MNT value close to 1 shows that the overall amount of bits transmitted over the radio interface is received by all the multicast receivers.

\section{B. Multicriteria Decision-Making Problem: TOPSIS Approach}

The method used in this paper for choosing the algorithm that provides the best trade-off among the considered metrics is represented by the technique for order performance by similarity to ideal solution known as TOPSIS [19]. TOPSIS is one of the most used methods for MCDM problems and is based on the idea that the chosen solution among different alternatives should have the shortest geometric distance from the positive ideal solution and the longest geometric distance from the negative ideal solution. In this work we use an extension of TOPSIS provided by [35], where the final evaluation of the different metrics is made by exploiting a similarity approach instead of closeness criteria. In addition, we do not use vertex method to calculate the distance between two fuzzy ratings but we use fuzzy similarity. The problem of selecting the best metrics among those investigated above can be approximated to a supplier selection problem in a supply chain (typical of the TOPSIS method). The MCMD problem can be described as follows:

(i) a set of $T$ decision-makers called $D=D_{1}, D_{2}, \ldots, D_{t}$ represented by the five environments is taken into considerations;

(ii) a set of $p$ possible suppliers called $A=A_{1}, A_{2}, \ldots, A_{p}$ are represented by the algorithms;

(iii) a set on $q$ criteria, $C=C_{1}, C_{2}, \ldots, C_{q}$, with which supplier performance are evaluated (CDR, ADR, JainIndex, MNT);

(iv) a set of performance ratings called $X=x_{i j}, i=$ $1,2, \ldots, m, j=1,2, \ldots, n$ are described accurately in [35].

Assuming that a decision group has $t$ decision-makers and that all fuzzy ratings and weights are trapezoidal fuzzy numbers $\hat{x_{i j t}}=\left(a_{i j t}, b_{i j t}, c_{i j t}, d_{i j t}, e_{i j t}\right)$ and $\hat{w}=\left(a_{i j 1}, b_{i j 2}\right.$, $\left.c_{i j 3}, d_{i j 4}, e_{i j 5}\right) ; i=1,2, \ldots, p, j=1,2, \ldots, q$, respectively. Then, the aggregate fuzzy ratings can be expressed as:

$$
\hat{x}_{i j}=\left(a_{i j}, b_{i j}, c_{i j}, d_{i j}, e_{i j}\right) \text {, }
$$

where

$$
\begin{aligned}
& a_{i j}=\min _{t}\left\{a_{i j t}\right\} b_{i j}=\frac{1}{T} \sum_{t=1}^{T} b_{i j t}, \\
& c_{i j}=\frac{1}{T} \sum_{t=1}^{T} c_{i j t} d_{i j}=\max _{T}\left\{d_{i j t}\right\} .
\end{aligned}
$$

Hence, the aggregate fuzzy weights of each criterion can be calculated as:

$$
\hat{w}_{j}=\left(w_{j 1}, w_{j 2}, w_{j 3}, w_{j 4}, w_{j 5}\right),
$$

Therefore, the metrics-selection problem can be expressed in matrix form as:

$$
\begin{aligned}
\hat{X} & =\left[\begin{array}{cccc}
\hat{x}_{11} & \hat{x}_{12} & \cdots & \hat{x}_{1 q} \\
\hat{x}_{21} & \hat{x}_{22} & \cdots & \hat{x}_{2 q} \\
\vdots & \vdots & & \vdots \\
\hat{x}_{m 1} & \hat{x}_{p 2} & \cdots & \hat{x}_{p q}
\end{array}\right] \\
\hat{W} & =\left(w_{1}, w_{2}, w_{3}, w_{4}, w_{5}\right) .
\end{aligned}
$$

Since the set of criteria can be divided into benefit criteria (B) and cost criteria (C), the normalized fuzzy decision matrix can be represented as:

$$
\hat{R}=\left[\hat{r}_{i j}\right]_{p \times q}
$$

where the term $r_{i j}$ include both the set of benefit and cost criteria represented by $B$ and $C$, respectively. In order to preserve the trapezoidal property of the fuzzy numbers, the value of $r_{i j}$ is calculated differently based on the fact that the criteria is a benefit or a cost as follows:

$$
\begin{aligned}
& \hat{r}_{i j}=\left(\frac{a_{i j}}{d_{j}^{+}}, \frac{b_{i j}}{d_{j}^{+}}, \frac{c_{i j}}{d_{j}^{+}}, \frac{d_{i j}}{d_{j}^{+}}, \frac{e_{i j}}{d_{j}^{+}}\right), \quad j \in B, \\
& \hat{r}_{i j}=\left(\frac{a_{j}^{-}}{a_{i j}}, \frac{a_{j}^{-}}{b_{i j}}, \frac{a_{j}^{-}}{c_{i j}}, \frac{a_{j}^{-}}{d_{i j}}, \frac{a_{j}^{-}}{e_{i j}}\right), \quad j \in C,
\end{aligned}
$$

The weighted normalized fuzzy decision matrix by considering the importance of each criterion can be summarized as follows:

$$
\hat{V}=\left(\hat{v}_{i j}\right)_{p \times q}
$$

where $\hat{v}_{i j}=\hat{r}_{i j}(\cdot) \hat{w}_{j}$. According to the aim of the TOPSIS algorithm, the fuzzy ideal solution (FPIS, $\mathrm{A}^{+}$) and the fuzzy negative-ideal solution (FNIS, $\mathrm{A}^{-}$) need to be defined as:

$$
\begin{aligned}
& A^{+}=\left(\hat{v}_{1}^{+}, \hat{v}_{2}^{+}, \ldots, \hat{v}_{p}^{+}\right) \\
& A^{-}=\left(\hat{v}_{1}^{-}, \hat{v}_{2}^{-}, \ldots, \hat{v}_{p}^{-}\right)
\end{aligned}
$$

Following this type of thinking one clear and simple intuitive proposal for the choice of fuzzy positive ideal would be simply the set of ones and for negative ideals a set of zeros. Finally, we can calculate the fuzzy similarity matrix and, subsequently, simply compute the average of the similarities and use this as a similarity measure to make the ranking as:

$$
S_{i}^{+}=\frac{1}{q} \sum_{j=1}^{q} S_{v}\left(\hat{v}_{i j}, \hat{v}_{j}^{+}\right) .
$$

\section{Obtained Results}

1) Open Environment: As shown in Fig. 3(a), the Open environment represents the environment where multicast users experience the highest channel quality conditions. By focusing on the CDR plotted in Fig. 3(a), we can observe that in this environment the CDR of CMS is $3.6 \mathrm{Mbps}$, on average. The MLA and the subgroup-based schemes overcome the 


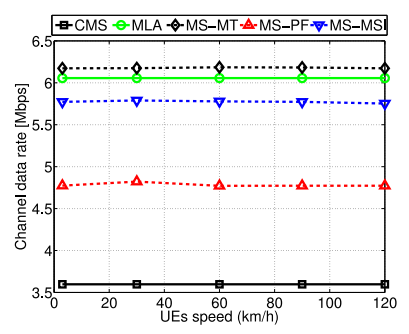

(a) Channel Data Rate

Fig. 3. Open environment.

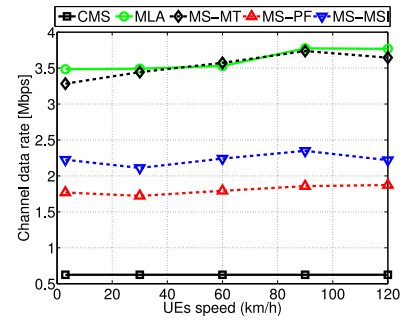

(a) Channel Data Rate

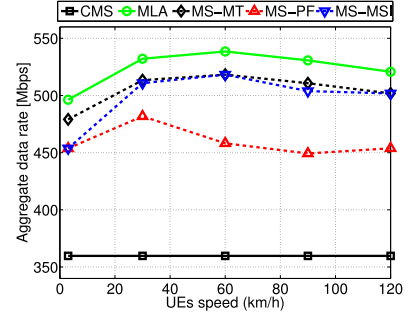

(b) Aggregate Data Rate

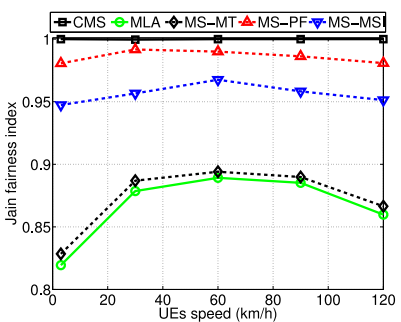

(c) Jain's Fairness Index

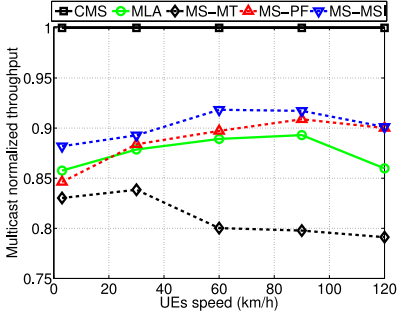

(d) Multicast Normalized Throughput

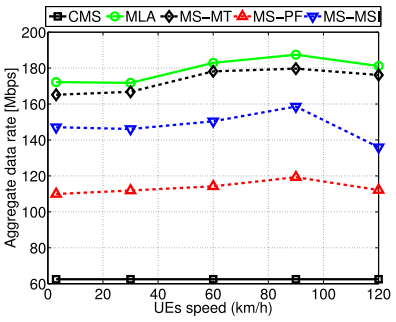

(b) Aggregate Data Rate

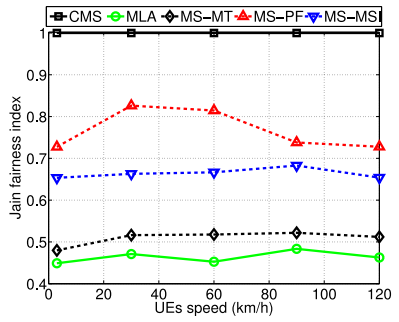

(c) Jain's Fairness Index

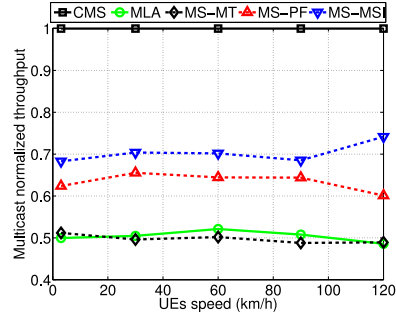

(d) Multicast Normalized Throughput

Fig. 4. Suburban environment.

limitations of CMS. In particular, the MLA and the MS-MT approaches obtain almost the same CDR, equal to $6 \mathrm{Mbps}$, on average, while the MS-PF policy achieves a performance of 4.9 Mbps. Finally, the new approach based on MS-MSI shows a CDR equal to $5.7 \mathrm{Mbps}$, on average, i.e., the MS-MSI increases the CDR by about $12 \%$ compared to the MS-PF.

Similarly to the CDR performance, the ADR results (depicted in Fig. 3(b)) show that the CMS is the worst performing policy, with an ADR of about $358 \mathrm{Mbps}$, on average. It is worth noticing that the trend of some considered metrics (i.e., the ADR) for some approaches could increase up to a given speed (i.e., $60 \mathrm{kmph}$ ). Then, the trend decreases when the users' mobility is further incremented (i.e., speed $>$ $60 \mathrm{kmph})$. The motivation of this behavior is due to the fact that in a satellite scenario for very low speeds the channel remains almost unchanged. In contrast, by increasing the speed, the channel conditions vary with a possible performance improvement. Finally, when users start to move with a considerable speed, the possibility that they experience worst channel conditions becomes higher. Therefore, the high intensity of mobility affects strongly the user performance. As we can observe, the MS-MT achieves a data rate close to the MS-MSI, i.e., the highest value is around $520 \mathrm{Mbps}$. The ADR of MS-PF, instead, decreases already when the speed is around $30 \mathrm{kmph}$ and the highest value achieved is $480 \mathrm{Mbps}$. Finally, the MLA outperforms the compared approaches by reaching an ADR of $538 \mathrm{Mbps}$ when the speed of the users is $60 \mathrm{kmph}$.

We now focus on the JFI, shown in Fig. 3(c). Being based on the single-rate approach, the CMS reaches the highest JFI, i.e., 1 , since all multicast members are served with the same data rate. In contrast, the MLA and the MS-MT achieve the poorest performance whereas the JFI of MS-PF results around 0.98 . Finally, MS-MSI reaches a value of 0.97 by showing that our proposed approach can obtain a JFI value close to the MS-PF with a mismatch of about $3 \%$, on average.

The results in terms of MNT are shown in Fig. 3(d). It is interesting to note that the MLA and the MS-MT have the lowest MNT, whereas CMS performs the best. This shows that although these policies are well designed in order to improve the throughput of multicast users, they do not reach an efficient spectrum utilization. Indeed, as also demonstrated by the poor performance in terms of JFI, the improvements of throughput values are only attained by a small amount of multicast destinations and, as a consequence, the spectrum is not efficiently exploited since a large portion of transmitted bits are received by only a limited set of users. The MNT of MS-PF is equal to 0.9 , on average, while this is equal to 0.93 for MSMSI. These results show that the MS-MSI can achieve a better radio channel exploitation compared to other subgroup-based policies.

2) Suburban Environment: In the Suburban environment, the channel quality of users experiences a degradation with respect to the Open environment. This involves a reduction in the performance of multicast members and, in general, of all considered policies. Indeed, if we focus for instance on the CDR shown in Fig. 4(a), we can observe that in this environment the CDR of CMS is 0.6 Mbps. Again, the MLA and the MS-MT approaches obtain the best performance whereas the MS-PF policy achieves a performance of about $1.7 \mathrm{Mbps}$. Finally, the MS-MSI shows a CDR equal to $2.3 \mathrm{Mbps}$, on average. In the Suburban environment, the MS-MSI increases the CDR of about $30 \%$ compared to the MS-PF. 


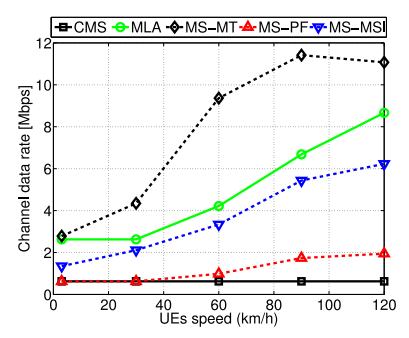

(a) Channel Data Rate

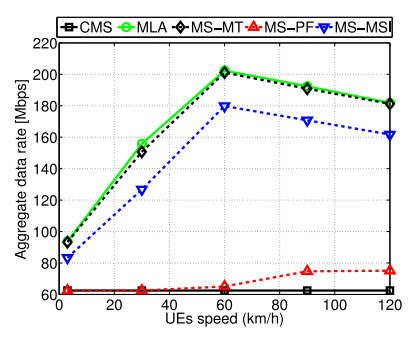

(b) Aggregate Data Rate

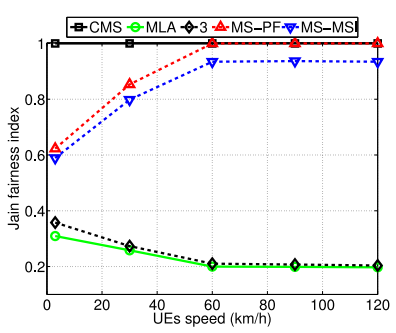

(c) Jain's Fairness Index

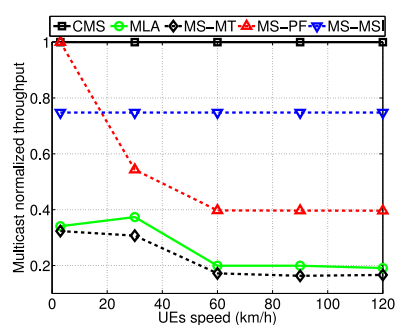

(d) Multicast Normalized Throughput

Fig. 5. Urban environment.

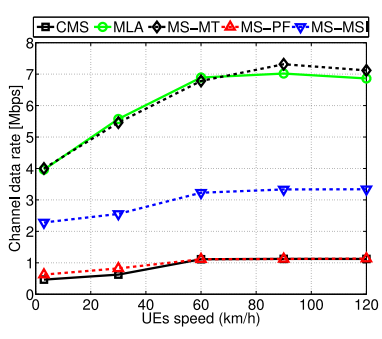

(a) Channel Data Rate

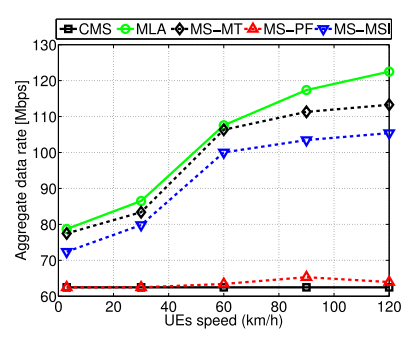

(b) Aggregate Data Rate

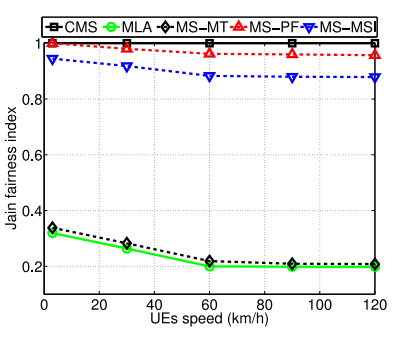

(c) Jain's Fairness Index

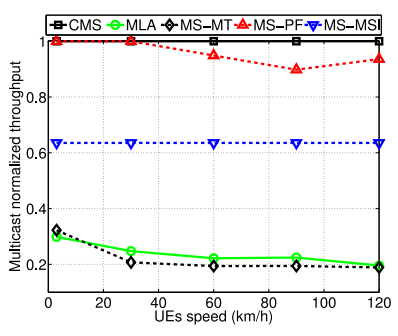

(d) Multicast Normalized

Fig. 6. Intermediate Tree Shadowed environment.

The ADR results (shown in Fig. 4(b)) show a similar trend compared to the CDR. In such a case, we can observe a decrease in the performance when the speed of the users is above $90 \mathrm{kmph}$. The CMS has the poorest ADR, equal to $62.5 \mathrm{Mbps}$, on average, while it reaches the maximum value of 180 and $188 \mathrm{Mbps}$, for the MS-MT and the MLA, respectively. The ADR of MS-PF is equal to $118 \mathrm{Mbps}$, on average, while MS-MSI reaches a performance of $160 \mathrm{Mbps}$ with a speed of $90 \mathrm{kmph}$, i.e., a gain of about $28 \%$ compared to the MS-PF.

The performance in terms of JFI is shown in Fig. 4(c). As expected, the CMS has a JFI equal to 1 . It is worth noting that when the channel conditions of multicast users become poorer, the JFI of MLA and MS-MT drastically decreases whereas the JFI of MS-PF decreases down to 0.75. The MS-MSI approach, instead, reaches a value of 0.7 , on average.

The results in terms of MNT are shown in Fig. 4(d). Again, the MLA and the MS-MT have the lowest MNT, i.e., 0.5, on average, while the MNT of MS-PF and MS-MSI is equal on average to 0.65 and 0.7 , respectively.

3) Urban Environment: Focusing on the Urban environment, we can observe that the performance of considered algorithms decreases, although we can always note that the behaviour of considered policies do not change. Indeed, by focusing on the CDR plotted in Fig. 5(a), we can observe that the CDR of CMS is $0.6 \mathrm{Mbps}$, on average. The MSMT overcomes the other considered approach whereas the MLA increases their CDR up to $8.8 \mathrm{Mbps}$. It is worth noting the behaviour of MS-PF, which has almost the same performance of CMS in the case of $3 \mathrm{kmph}$ and $30 \mathrm{kmph}$, while it reaches $2.3 \mathrm{Mbps}$ with a user speed equal to $120 \mathrm{kmph}$. In any case, it is observed that the MS-PF is hardly influenced

by the radio propagation environment and by the user mobility speed. Finally, the MS-MSI shows a CDR that varies from 1.3 to $6 \mathrm{Mbps}$.

A similar trend can be found in the ADR results, shown in Fig. 5(b). It is worth noting that, except for the CMS and MS-PF, all considered policies show a reduction in terms of ADR when the users' mobility is above $60 \mathrm{kmph}$. Anyway, the relationship among evaluated schemes does not change. Indeed, the MLA and MS-MT algorithms show the best performance and almost the same trend, while the CMS has the poorest ADR, i.e., $62 \mathrm{Mbps}$. The ADR of MS-PF ranges from $62 \mathrm{Mbps}$ to $78 \mathrm{Mbps}$. Finally, the MS-MSI achieves an ADR that varies from 80 to $180 \mathrm{Mbps}$.

As for the Suburban environment, the performance in terms of JFI (Fig. 5(c)) shows that the CMS achieves a JFI equal to 1 whereas the values of the MLA and MS-MT drastically decrease due to the lower channel conditions of the multicast users (i.e., given by the higher users speed). In this case, the JFI of the MS-MSI is close to the MS-PF and it increases with the speed until it reaches a JFI value close to 1 (i.e., speed equal or more to $60 \mathrm{kmph}$ ). Concerning the MNT (Fig. 5(d)), it is important to observe that the MS-MSI attains a performance equal to 0.75 in all considered cases. The MNT of MS-PF decreases from 1 to 0.4, while the one of MLA and MS-MT varies from 0.3 to 0.2 .

4) Intermediate Tree Shadowed Environment: The results attained in the Intermediate Tree Shadowed environment follow the same behaviour of those in the Urban environment, i.e., the results attained by the different considered scheme are influenced by the users' mobility speed and decrease when the speed increases. In addition, in this environment we can further observe that the performance of MS-PF becomes close 


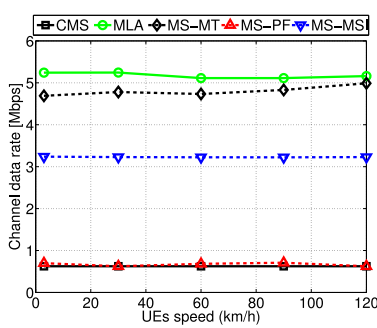

(a) Channel Data Rate

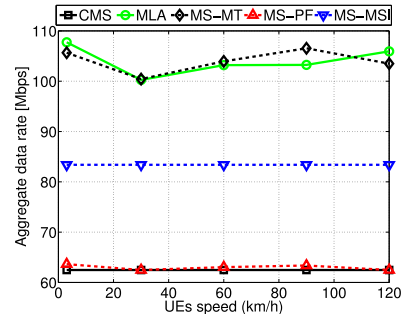

(b) Aggregate Data Rate

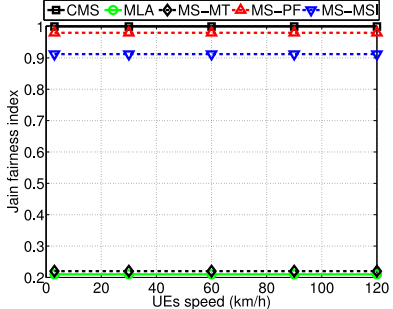

(c) Jain's Fairness Index

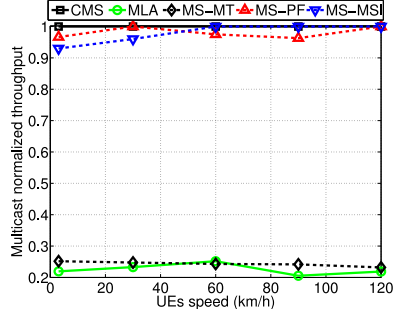

(d) Multicast Normalized Throughput

Fig. 7. Heavy Tree Shadowed environment.

TABLE VI

Example of Decision Matrices From the Decision MaKers Considering 3kmph

\begin{tabular}{cccccc}
\hline Criteria & $D_{1}$ & $D_{2}$ & $D_{3}$ & $D_{4}$ & $D_{5}$ \\
\hline CDR & (VP G G VP F) & (VP F F VP MP) & (MG VG VG MG G) & (VP MG MG P F) & (VP MP MP VP P) \\
\hline ADR & (VP MP MP P MP) & (VP P P VP P) & (F G G G G) & (VP MP MP P MP) & (VP P P VP P) \\
\hline JI & (VG P P VG VG) & (VG P P VG VG) & (VG G G VG VG) & (VG F F MG MG) & (VG P P VG VG) \\
\hline GP & (VG P P VG F) & (VG P P VG MG) & (VG G G G VG) & (VG F F F F) & (VG P P VG MG) \\
\hline
\end{tabular}

to the CMS. For instance, if we consider the CDR results (plotted in Fig. 6(a)), the performance of CMS is equal to 0.9 Mbps while the one of MS-PF is equal to 0.95, on average. This means that, in environments where multicast devices are in bad channel conditions, the MS-PF optimization problem for multicast subgroup formation is not able to exploit the multi-user diversity. On the contrary, the CDR of MLA and MS-MT varies from 4 to $7 \mathrm{Mbps}$, on average. Finally, the CDR of MS-MSI is in the range from 2.3 to $3.4 \mathrm{Mbps}$.

The ADR results, shown in Fig. 6(b), reflect those in terms of CDR. Indeed, the CMS and the MS-PF achieve similar values, i.e., 62.5, on average. The ADR of MLA varies from 78 and $120 \mathrm{Mbps}$ whereas MS-MT varies from 78 and $110 \mathrm{Mbps}$. Finally, the MS-MSI obtain a result which varies from 73 to 105 Mbps.

It is interesting to discuss about the MNT figures, depicted in Fig. 6(d). Indeed, the CMS achieves a performance equal to 1 in each considered case, while the one of MS-MSI is equal to 0.64 . The result of other policies is not strongly affected by the users' speed. For the MS-PF, the MNT varies from 1 to 0.90 , while for other policies it decreases from 0.3 to 0.2 .

5) Heavy Tree Shadowed Environment: We now consider the Heavy Tree Shadowed environment, which is the worst environment in terms of channel conditions. We can observe that the MS-PF policy in this environment achieves almost the same results of the CMS, and this is more evident compared to the previous Intermediate Tree Shadowed environment. Indeed, if we consider the CDR in Fig. 7(a), the CMS has a performance equal to $0.6 \mathrm{Mbps}$, while the one of MS-PF is 0.62 , on average. In addition, it is worth noting that the performance of the considered policies in this environment is not strongly influenced by the users' mobility speed. Indeed, the results do not vary meaningfully at different evaluated speeds. For instance, the CDR of MLA and MS-MT are close to 5 Mbps for each case, while the MS-MSI achieves a CDR equal to $3.2 \mathrm{Mbps}$, on average.
The ADR (shown in Fig. 7(b)) of CMS and MS-PF is equal to 62 and $63 \mathrm{Mbps}$, respectively, while it is equal to $105 \mathrm{Mbps}$ (on average) for the MLA and the MS-MT. The MS-MSI achieves an ADR equal to $84 \mathrm{Mbps}$, on average, and this means that it introduces a gain of about $33 \%$ compared to the MS-PF.

Concerning the MNT, shown in Fig. 7(d), we can observe that the CMS has again the MNT equal to 1 in each environment. Being close to the behaviour of CMS, the MS-PF achieves a MNT of about 0.95 , while the proposed MS-MSI shows a performance around 0.98. Finally, the MLA and MS-MT policies have a MNT equal to 0.2 , on average.

\section{TOPSIS Results}

In order to establish which of the subgrouping approaches provide the most suitable solution to solve the metrics decision problem, we consider one of the most used multi-decision solver named TOPSIS. In particular, to model the MCDM problem, we consider $D=5$ decision-makers represented by the different environments and $n=4$ criteria represented by the considered parameters (i.e., CDR, ADR, MNT and FI) to compare the approaches (i.e., CMS, MLS, MS-MT, MS-PF, MS-MSI). A weight has been assigned to each metric by following the linguistic variables expressed in positive fuzzy number proposed in [35]. ${ }^{2}$ Therefore, the TOPSIS algorithm has been executed for each of the considered speeds (i.e., 3, $30,60,90,120 \mathrm{kmph}$ ) and a final ranking is created in order to decide which is the best metric that could be used.

As an example, Table VI shows the values assigned by the different decision makers by considering only a speed of $3 \mathrm{kmph}$. Obviously, similar tables (not shown in this paper for the lack of space) have been obtained for the further considered user speeds. Therefore, every step of the TOPSIS method is executed through a Matlab simulator. The results shown in

\footnotetext{
${ }^{2} \mathrm{VP}=$ Very Poor; $\mathrm{P}=$ Poor; $\mathrm{MP}=$ Medium Poor; $\mathrm{F}=$ Fair; $\mathrm{MG}=$ Medium Good; $\mathrm{G}=$ Good; VG = Very Good
} 
TABLE VII

EXAmple of DeCision MATRICES From the DECISION MAKERS CONSIDERING 60kmph

\begin{tabular}{cccccc}
\hline Speed & CMS & MLA & MS-MT & MS-PF & MS-MSI \\
\hline $3 \mathrm{kmph}$ & 0.4015 & 0.3565 & 0.5191 & 0.5005 & 0.6302 \\
\hline $30 \mathrm{kmph}$ & 0.4154 & 0.3564 & 0.5207 & 0.4858 & 0.6145 \\
\hline $60 \mathrm{kmph}$ & 0.4294 & 0.3711 & 0.5251 & 0.4756 & 0.6276 \\
\hline $90 \mathrm{kmph}$ & 0.4145 & 0.3678 & 0.5256 & 0.4712 & 0.6234 \\
\hline $120 \mathrm{kmph}$ & 0.4234 & 0.3712 & 0.5331 & 0.4845 & 0.6389 \\
\hline
\end{tabular}

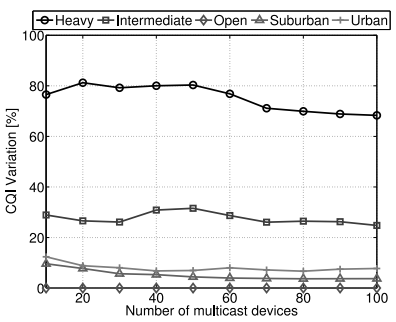

(a) $3 \mathrm{kmph}$

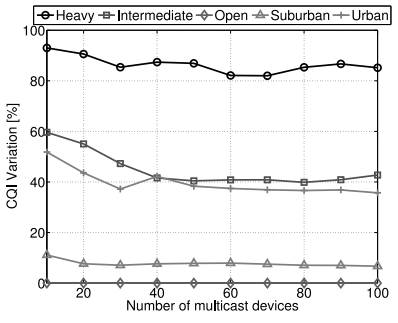

(b) $120 \mathrm{kmph}$
Fig. 8. Variation of user' CQI for $3 \mathrm{kmph}$ and $120 \mathrm{kmph}$.

Table VII allow us to clearly assert that the proposed MS-MSI metric provides the best performance results for subgroup formation compared to the other four approaches analyzed in this work. Hence, it allows to provide the best performance trade-off among the considered criteria.

\section{E. Delay Impact Analysis}

The last analysis conducted in this work is focused on the impact of satellite propagation delay to the performance of considered multicast policies. Delay is defined according the definition given in the previous sections. In particular, this analysis aims to demonstrate the robustness to delay of subgrouping-based approach. In Fig. 8 the misalignment between the channel quality feedback transmitted to the S-eNodeB (i.e., CQI) and the channel quality experienced by the mobile users at the reception of data traffic (hereinafter named CQI misalignment) is reported for the five environments and the two speeds (i.e., 3 and $120 \mathrm{kmph}$ ). Therefore, Fig. 8 provides an example of CQI variation during one round trip time (RTT, approximately $0.54 \mathrm{sec}$ ) for a generic user in each considered environment. We can observe that the higher the speed, the higher is the CQI variation during the RTT. It is worth noticing that the reported results do not depend on the particular RRM implementation whereas they do depend on the satellite delay and according to the different environments. As expected, in the heavy tree shadowed and intermediate tree shadowed environments the delay introduced by satellite link heavily influences the CQI misalignment, which does not significantly changes in the open and suburban environments.

Fig. 9 depicts the analysis on the percentage of multicast members with a BLER lower than $10 \%$. This metric measures the percentage of users that are not able to successfully demodulate the data received by the satellite. This parameter is useful to show the impact of the large propagation delay on the user's session quality, since the CQI of multicast devices

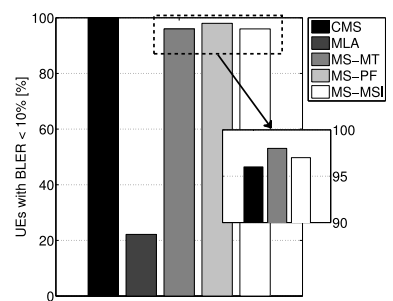

(a) $3 \mathrm{kmph}$

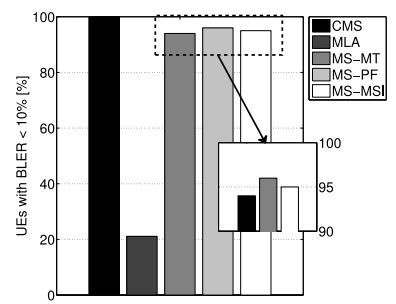

(b) $120 \mathrm{kmph}$
Fig. 9. \# UEs with BLER $<10 \%$ for the Heavy Tree Shadowed environment.

could vary from when it is transmitted to the S-eNodeB to when multicast data is transmitted.

Due to the length constraints on this paper, we reported only on the behaviour related to the Heavy Tree Shadowed, as it presents the highest CQI misalignment with respect the other environments. As shown in Fig. 9, both CMS and subgrouping schemes obtain a performance very close to $100 \%$, i.e., almost every multicast user is able to successfully demodulate the received data. Obviously, CMS presents the highest robustness to the delay because it always transmits with the lowest MCS, hence the user channel variations during the round trip time do not affect the BLER because the transmission parameters are always tuned to the user with the worst channel conditions. Therefore, even if the CQI of the users changes during the round trip time, all the users will be able to correctly download the content (100\% of users receives data with a BLER $<10 \%$, see Fig. 9).

For similar reasons, this subgrouping approach is robust to long propagation delays, because in each multicast subgroup, users are managed with a conservative approach (i.e., similar to CMS); therefore, inside every subgroup, users are served by exploiting a transmission parameters experienced by the user with the worst channel conditions. Performance degradation (in terms of BLER, and so on) related to the satellite round trip time happens only for those users that experience the following twofold conditions: ( $i$ ) a worsening of the channel conditions; (ii) a CQI lower than the CQI of their own sub-group. This rarely happens, indeed, about the $97 \%$ of users receive data with a BLER $<10 \%$, see Fig. 9. In all the other situations (i.e., (i) the CQI increases during the long propagation delay, by also changing the subgroup, (ii) the CQI decreases but remains greater than the CQI of the own sub-group) the satellite users will correctly receive the data.

On the other hand, by considering the MLA, almost $21 \%$ of multicast users are able to receive the data during the multicast session. This is due to the fact that the opportunistic approaches (i.e., MLA), which provide meaningful performance improvement in terrestrial environment, suffer of the delay introduced by satellite systems. Indeed, such approaches serve in every TTI only a portion of users with the aim of maximizing a given cost function (i.e., the ADR in MLA). Therefore, if the channel quality of users decreases during the RTT, a large amount of users will not be able to decode correctly the multimedia content. In fact, the percentage of the users with a BLER value less than $10 \%$ of the MLA approach varies from a percentage of $80 \%$, in case of 
TABLE VIII

TERrestrial vs. SATELLITE CoMPARISON

\begin{tabular}{c|cc|cc} 
& \multicolumn{2}{|c|}{ MLA } & \multicolumn{2}{c}{ MS-MSI } \\
& LTE & S-LTE & LTE & S-LTE \\
\hline \hline ADR & 218.93 & 105.93 & 175.16 & 84.97 \\
\hline \# UEs BLER $<10 \%$ & 100 & 21 & 100 & 97 \\
\hline MNT & 0.48 & 0.22 & 1 & 1 \\
\hline \hline
\end{tabular}

Open environment, to a value of $21 \%$ by considering the most delay influenced environment (represented by the Heavy Tree Shadowed environment). This implies an increased number of retransmission requests, which are not easy to manage in a satellite environment.

\section{F. Comparison With Terrestrial Systems}

In previous studies [15] (and our referred works) we demonstrated that multicast subgrouping schemes are suitable for improving the performance of the terrestrial networks and allow to increase the percentage of users served with a considerable data rate in the same TTI. Moreover, in [15] we also demonstrated that opportunistic approaches (i.e., Opportunistic Multicast Scheduling, OMS) allow to provide the highest data rate. Both the approaches in the terrestrial environment do not suffer of retransmission issues because typically the BLER is less than $10 \%$. Nevertheless, it is not a natural consequence that the results obtained for the terrestrial system will remain the same also for the satellite environment. In fact, the long propagation delay and the diverse channel characterization (i.e., typical of the satellite transmissions) pose considerable differences between the two systems.

For the sake of completeness, in this section we provide a comparison between MLA and MS-MSI for terrestrial and satellite environments in terms of (i) aggregate data rate, (ii) multicast normalized throughput, and (iii) \# UE with BLER < $10 \%$. We want to demonstrate that the subgrouping approach is able to maintain the same performance achieved for terrestrial systems, whereas the opportunistic approach (i.e., MLA) cannot be exploited for multimedia content delivery in satellite environments because it could require a high number of retransmissions.

The simulation campaign has been performed by considering $K=100$ users, a speed equal to $120 \mathrm{kmph}$, and the Heavy Tree Shadowed environment, as it presents the highest CQI misalignment with respect to the other environments. Obviously, we exploited the Pérez-Fontán channel model for the satellite environment, which is considerably different to the standard LTE channel model for the terrestrial systems. The obtained results are shown in Table VIII.

The 50\% reduction of ADR in both the algorithms depends on the different channel models. More interesting results have been obtained in terms of \# of UE with BLER $<10 \%$ and MNT. In particular for MLA, only $21 \%$ of the users are able to decode correctly the message (i.e., the $79 \%$ of users experience a BLER higher than 10\%) whereas for the MS-MSI this value keeps almost unchanged. Similarly, the MNT in case of MLA has a reduction of around $50 \%$. It means that only a small percentage of users are served with a data rate that is close to the maximum amount of bits that can be transmitted with a given channel quality (i.e., please refer to the CDR metric in our paper). It is worth noticing that in both terrestrial and satellite environments the MS-MSI approach has the same behaviour in terms of MNT. In conclusion, we can assert that the opportunistic approach is strongly affected by the long propagation delay, whereas the subgrouping approach is robust to the CQI misalignment due to the long RTT (i.e., $0.54 \mathrm{sec}$ ).

\section{CONCLUSION}

In this paper, we focused on RRM techniques for providing multimedia content in emerging 5G-satellite networks. A LTE satellite system architecture has been designed for supporting the evolved multicast/broadcast multimedia service and different multicast policies have been analyzed and compared with the proposed multicast subgrouping-maximum satisfaction index (MS-MSI) algorithm. By adopting a multi-criteria decision-making solver based on TOPSIS, we demonstrated through an exhaustive simulation campaign that the proposed MS-MSI approach provides the best overall performance and overcomes the limitations of the previous techniques proposed in the literature, such as the robustness to the long propagation delays that in satellite environments play an important role. Furthermore, we demonstrated that our subgrouping approach provides good results for both satellite and terrestrial environments. This gives us confidence that this approach could be efficiently exploited in integrated satellite-terrestrial systems where the satellite will play an important role in the emerging 5G networks for offloading the terrestrial traffic and for providing multimedia contents. Moreover, in a future $5 \mathrm{G}$ scenario, some smart devices could be used as the gap filler in Digital Video Broadcasting systems. In particular, such smart devices in a cell could simultaneously transmit the same signal received from the S-LTE to their D2D-connected devices for forwarding the S-LTE services to their shadowed users. The D2D-receivers could consider these replications as multipath components of the same signal.

\section{REFERENCES}

[1] NetWorld2020 White Paper, "SatCom WG: The role of satellites in 5G," Jul. 2014. [Online]. Available: http://networld2020.eu/white-papers/

[2] R. Khan, "System and method for satellite-long term evolution (S-LTE) air interface," U.S. Patent 12/209 436, 2008.

[3] S. Liu, F. Qin, Z. Gao, Y. Zhang, and Y. He, "LTE-satellite: Chinese proposal for satellite component of IMT-advanced system," China Commun., vol. 10, no. 10, pp. 47-64, Oct. 2013.

[4] A. Asadi, Q. Wang, and V. Mancuso, "A survey on device-to-device communication in cellular networks," IEEE Commun. Surveys Tuts., vol. 16, no. 4, pp. 1801-1819, Dec. 2014.

[5] S. Andreev, A. Pyattaev, K. Johnsson, O. Galinina, and Y. Koucheryavy, "Cellular traffic offloading onto network-assisted device-to-device connections," IEEE Commun. Mag., vol. 52, no. 4, pp. 20-31, Apr. 2014.

[6] L. Wang and $\mathrm{H}$. Wu, "Fast pairing of device-to-device link underlay for spectrum sharing with cellular users," IEEE Commun. Lett., vol. 18 , no. 10, pp. 1803-1806, Oct. 2014.

[7] A. Hava, Y. Ghamri-Doudane, G.-M. Muntean, and J. Murphy, "Increasing user perceived quality by selective load balancing of video traffic in wireless networks," IEEE Trans. Broadcast., vol. 61, no. 2, pp. 238-250, Jun. 2015.

[8] Cisco. (Sep. 2014). Cisco VNI Forecast Widget 2014. [Online]. Available: http://www.ciscovni.com/forecast-widget/advanced.html 
[9] Cisco Visual Networking Index: Forecast and Methodology, 2013-2018, Cisco, San Jose, CA, USA, Jun. 2014

[10] 3GPP, "General aspects and principles for interfaces supporting multimedia broadcast multicast service (MBMS) within E-UTRAN. Release 11," Tech. Rep. 3GPP TS 36.440, Sep. 2012.

[11] A. Tassi, I. Chatzigeorgiou, and D. Vukobratovic, "Resource-allocation frameworks for network-coded layered multimedia multicast services," IEEE J. Sel. Areas Commun., vol. 33, no. 2, pp. 141-155, Feb. 2015.

[12] A. Sali, H. A. Karim, G. Acar, B. Evans, and G. Giambene, "Multicast link adaptation in reliable transmission over geostationary satellite networks," Wireless Pers. Commun., vol. 62, no. 4, pp. 759-782, 2012.

[13] G. Aiyetoro, G. Giambene, and F. Takawira, "Link adaptation in satellite LTE networks," J. Adv. Inf. Technol., vol. 5, no. 1, pp. 37-43, 2014.

[14] G. Araniti, M. Condoluci, and A. Petrolino, "Efficient resource allocation for multicast transmissions in satellite-LTE networks," in Proc. IEEE Glob. Commun. Conf. (GLOBECOM), Atlanta, GA, USA, Dec. 2013 , pp. 3023-3028.

[15] G. Araniti et al., "Evaluating the performance of multicast resource allocation policies over LTE systems," in Proc. IEEE Int. Symp. Broadband Multimedia Syst. Broadcast. (BMSB), Ghent, Belgium, Jun. 2015, pp. 1-6.

[16] R. O. Afolabi, A. Dadlani, and K. Kim, "Multicast scheduling and resource allocation algorithms for OFDMA-based systems: A survey," IEEE Commun. Surveys Tuts., vol. 15, no. 1, pp. 240-254, Mar. 2013.

[17] S. Mukherjee et al., "Mode switching algorithms for DVB-S2 links in W band," in Proc. IEEE Glob. Telecommun. Conf. (GLOBECOM), Miami, FL, USA, Dec. 2010, pp. 1-5.

[18] 3GPP, "Evolved universal terrestrial radio access (E-UTRA): Physical layer procedures. Release 11," Tech. Rep. 3GPP TS 36.213, Sep. 2012.

[19] H. Ching-Lai, L. Young-Jou, and L. Ting-Yun, "A new approach for multiple objective decision making," Comput. Oper. Res., vol. 20, no. 8 , pp. 889-899, Oct. 1993.

[20] E. Timmerman, "An approach to vendor performance evaluation," IEEE Eng. Manag. Rev., vol. 15, no. 3, pp. 14-20, Sep. 1987.

[21] R. E. Gregory, "Source selection: A matrix approach," J. Purchasing Mater. Manag., vol. 22, no. 2, pp. 24-29, 1986.

[22] R. L. Nydick and R. P. Hill, "Using the analytic hierarchy process to structure the supplier selection procedure," Int. J. Purchasing Mater. Manag., vol. 28, no. 2, pp. 31-36, 1992.

[23] J. Sarkis and S. Talluri, "A model for strategic supplier selection," J. Supply Chain Manag., vol. 38, no. 4, pp. 18-28, Dec. 2002.

[24] J. Jiang, "Channel quality indictor (CQI) correcting method and device in LTE emission mode 7," China Patent CN 101753190 A, Jun. 2010.

[25] J. Kim and M. Kim, "ARMA prediction of SBAS ephemeris and clock corrections for low earth orbiting satellites," Int. J. Aerosp. Eng., vol. 2015, 2015, Art. ID 165178. [Online]. Available: http://dx.doi.org/10.1155/2015/165178

[26] ETSI, "Satellite earth stations and systems (SES); advanced satellite based scenarios and architectures for beyond 3G systems, v.1.1.1," Tech. Rep. TR 102 662, Mar. 2010.

[27] M. De Sanctis et al., "Waveform design solutions for EHF broadband satellite communications," IEEE Commun. Mag., vol. 53, no. 3, pp. 18-23, Mar. 2015

[28] C. Suh and J. Mo, "Resource allocation for multicast services in multicarrier wireless communications," IEEE Trans. Wireless Commun., vol. 7, no. 1, pp. 27-31, Jan. 2008.

[29] F. P. Fontán, M. Vázquez-Castro, C. E. Cabado, J. P. Garcia, and E. Kubista, "Statistical modeling of the LMS channel," IEEE Trans. Veh. Technol., vol. 50, no. 6, pp. 1549-1567, Nov. 2001.

[30] H. Smith, S. K. Barton, J. G. Gardiner, and M. Sforza, "Characterization of the land mobile-satellite (LMS) channel at L and S bands: Narrowband measurements," Bradford Univ., Bradford, U.K., Eur. Space Agency, Paris, France, Tech. Rep. 104 433/114 473, 1992.

[31] C. Loo, "A statistical model for a land mobile satellite link," IEEE Trans. Veh. Technol., vol. 34, no. 3, pp. 122-127, Aug. 1985.

[32] C. Suh and J. Mo, "Resource allocation for multicast services in multicarrier wireless communications," IEEE Trans. Wireless Commun., vol. 7, no. 1, pp. 27-31, Jan. 2008.

[33] L. Militano, A. Iera, and F. Scarcello, "A fair cooperative content-sharing service," Comput. Netw., vol. 57, no. 9, pp. 1955-1973, Jun. 2013.

[34] A. Iera, L. Militano, L. P. Romeo, and F. Scarcello, "Fair cost allocation in cellular-bluetooth cooperation scenarios," IEEE Trans. Wireless Commun., vol. 10, no. 8, pp. 2566-2576, Aug. 2011.

[35] P. Luukka, "Fuzzy similarity in multicriteria decision-making problem applied to supplier evaluation and selection in supply chain management," Adv. Artif. Intell., vol. 2011, Art. ID 353509, 2011. [Online]. Available: http://dx.doi.org/10.1155/2011/353509

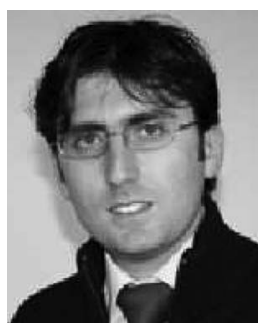

Giuseppe Araniti received the Laurea and $\mathrm{Ph} . \mathrm{D}$. degrees in electronic engineering from the Mediterranea University of Reggio Calabria, Italy, in 2000 and 2004, respectively. $\mathrm{He}$ is an Assistant Professor of Telecommunications with the Mediterranea University of Reggio Calabria. His major area of research includes personal communications systems, enhanced wireless and satellite systems, traffic and radio resource management, multicast and broadcast services, and device-to-device and machine type communications over $4 \mathrm{G} / 5 \mathrm{G}$ cellular networks.

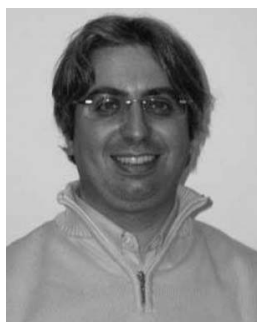

Igor Bisio received the Laurea degree in telecommunications engineering and the Ph.D. degree in information and communication sciences from the University of Genoa, Italy, in 2002 and 2006, respectively. He is currently an Assistant Professor with the University of Genoa. He has authored around 100 scientific papers, including international journals, international conferences, and book chapters. His main research activity concerns digital signal processing over smartphone platforms, contextawareness, Internet of things, and satellite systems. Since 2012, he has been the Chair of the IEEE Communications Society Satellite and Space Communications Technical Committee, and a member of the IEEE Internet of Things Special Issue Group of the IEEE Signal Processing Society since 2014. He is the Technical Committee Co-Chair of several international conferences, including the IEEE Globecom and ICC. He has organized special issues of the international journals and magazines.

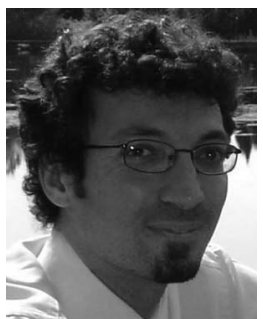

Mauro De Sanctis received the Laurea degree in telecommunications engineering and the $\mathrm{Ph} . \mathrm{D}$. degree in telecommunications and microelectronics engineering from the University of Roma Tor Vergata, Italy, in 2002 and 2006, respectively. He co-authored over 80 scientific papers, including international journals, international conferences, and book chapters. His main areas of interest are wireless terrestrial and satellite communication networks, human activity recognition, data mining, and information theory. He serves as an Associate Editor for the IEEE Aerospace and Electronic Systems Magazine.

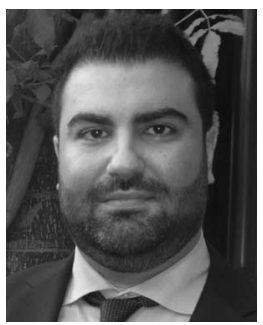

Antonino Orsino received the B.Sc. degree in telecommunication engineering from the Mediterranea University of Reggio Calabria, Italy, in 2009, and the M.Sc. degree from the University of Padova, Italy, in 2012. He is currently pursuing the Ph.D. degree with the DIIES Department, Mediterranea University of Reggio Calabria. His current research interests include device-to-device and machine-to-machine communications in $4 \mathrm{G} / 5 \mathrm{G}$ cellular systems. He has served as a Reviewer for several major IEEE conferences and journals.

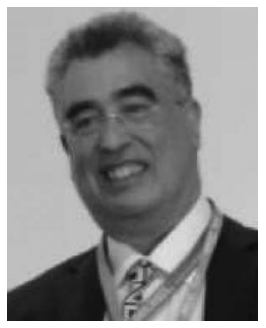

John Cosmas is currently a Professor of Multimedia Systems with the School of Engineering and Design, Brunel University, London, U.K. He coleads the Wireless Networks and Communications Research Centre. $\mathrm{He}$ is the Course Director of M.Sc. Advanced Multimedia Design and 3-D Technologies. Since 1986, he has participated in 11 EU-IST and two EPSRC-funded research projects, and has led CISMUNDUS, PLUTO, and 3-D MURALE. His current research interests include the development of multimedia systems applied to future of broadcasting, the future of Internet and 3-D multimedia video/graphics design, and the synergies between these technologies. He is an Associate Editor of the IEEE TRANSACTIONS ON BROADCASTING. 